\title{
Statistical properties of eigenstate amplitudes in complex quantum systems
}

\author{
Wouter Beugeling, ${ }^{1,2,3}$ Arnd Bäcker, ${ }^{1,4}$ Roderich Moessner, ${ }^{1}$ and Masudul Haque ${ }^{1,5}$ \\ ${ }^{1}$ Max-Planck-Institut für Physik komplexer Systeme, Nöthnitzer Straße 38, 01187 Dresden, Germany \\ ${ }^{2}$ Lehrstuhl für Theoretische Physik I \& II, Technische Universität Dortmund, Otto-Hahn-Straße 4, 44221 Dortmund, Germany \\ ${ }^{3}$ Physikalisches Institut, Universität Würzburg, Am Hubland, 97074 Würzburg, Germany \\ ${ }^{4}$ Technische Universität Dresden, Institut für Theoretische Physik and Center for Dynamics, 01062 Dresden, Germany \\ ${ }^{5}$ Department of Theoretical Physics, Maynooth University, Co. Kildare, Ireland
}

(Received 10 November 2017; revised manuscript received 20 June 2018; published 7 August 2018)

\begin{abstract}
We study the eigenstates of quantum systems with large Hilbert spaces, via their distribution of wave-function amplitudes in a real-space basis. For single-particle "quantum billiards," these real-space amplitudes are known to have Gaussian distribution for chaotic systems. In this work, we formulate and address the corresponding question for many-body lattice quantum systems. For integrable many-body systems, we examine the deviation from Gaussianity and provide evidence that the distribution generically tends toward power-law behavior in the limit of large sizes. We relate the deviation from Gaussianity to the entanglement content of many-body eigenstates. For integrable billiards, we find several cases where the distribution has power-law tails.
\end{abstract}

DOI: 10.1103/PhysRevE.98.022204

\section{INTRODUCTION}

Except for particularly simple systems, eigenstates of quantum Hamiltonians are complex objects, described by an exponentially large number of coefficients (amplitudes). Energy eigenstates are constitutive to the formulation of quantum mechanics. They are also essential in describing closed quantum systems, e.g., in considerations of thermalization [1-5]. Thus, one might reasonably regard the structure of eigenstates, e.g., the statistical properties of amplitudes, as being fundamental to our understanding of the quantum world. Amplitude distributions have been studied for single-particle (quantum billiard) systems [6-14]. However, little is known about corresponding distributions for quantum many-body Hamiltonians. In this work, we address distributions of coefficients (in the basis of real-space configurations), clarifying in particular the consequences of integrability.

While it is difficult to find a universally accepted definition of quantum integrability $[15,16]$, we will refer to systems with Poissonian level-spacing statistics (within a single symmetry sector) to be integrable or regular, and to those with random-matrix statistics as nonintegrable or chaotic. This distinction appears both in single-particle billiards $[17,18]$ and in many-body systems [19-30]. This operational definition is inadequate in some situations, but will suffice for this work. Many-body integrable systems include noninteracting ("free") fermions, free bosons, and systems solvable by Bethe ansatz. Integrable quantum billiards are those whose corresponding classical problems have as many independent conserved quantities as degrees of freedom. Some further comments on integrability are provided in Appendix A.

For quantum billiard systems, the distribution of real-space amplitudes $\psi(\vec{x})=\langle\vec{x} \mid \psi\rangle$ of eigenstates has been studied both for chaotic and for mixed systems [6-12,14,31]. In the chaotic case the amplitude distribution is expected to be Gaussian for almost all eigenstates (with some possible exceptions $[32,33]$ ).
This follows from the conjecture that high-energy eigenstates of chaotic billiards resemble random superpositions of many plane waves leading to a Gaussian distribution by the central limit theorem [7,34]. For single-particle systems, the particle position is the natural basis in which to express the amplitudes. In the many-body case, the choice of basis is less obvious, but a direct generalization is the basis of many-body configurations in real space. For lattice systems, this is also a widely used basis for numerical diagonalization. Our study focuses on coefficients in this basis.

For nonintegrable systems, we show that eigenstates away from spectral edges have Gaussian coefficient distributions. The resemblance to Gaussian form improves with increasing deviation from integrability, and also improves systematically with system size. For integrable many-body systems, there is clear deviation from Gaussian shape. We provide evidence that the distribution approaches a power law as the size is increased. The convergence is extremely slow; a meaningful scaling analysis could only be performed for free fermions, but data for several integrable systems show the same trend. An analytic argument is constructed for a toy model of distinguishable particles, which accounts for the power-law form and the slow convergence. The presented numerical data and arguments, taken together, naturally lead to the conjecture that eigenstates of integrable many-body systems generically have power-law coefficient distributions in the large-size ("thermodynamic") limit. This conjecture is remarkable because "generic" results are usually expected for chaotic rather than integrable systems.

We relate the coefficient distribution to the entanglement entropy between two spatial partitions. We show that larger deviations from Gaussian shape correlate strongly with low entanglement and provide intuition for this correlation.

We also present some results for integrable quantum billiard systems. Explicit calculation shows in a few cases that the amplitude distributions have power-law tails. An extended power-law region can appear when the regular eigenfunctions 
contain many inequivalent peaks. The feature is intriguing but is not present in all integrable billiard systems.

This article is structured as follows. In Sec. II, we introduce the many-body models and present a general study of their coefficient distributions, highlighting the differences between nonintegrable and integrable many-body systems, the deviation from Gaussianity and the correlation of this deviation with the entanglement entropy. In Sec. III, we focus on integrable many-body systems, and provide numerical evidence and argumentation supporting approach to power-law behavior in the large-size limit. In Sec. IV, we consider several single-particle quantum billiard systems and present results on amplitude distributions for several integrable and weakly nonintegrable billiards. Section V discusses the context and some implications of our results. We provide additional data and supporting discussions in the appendices.

\section{MANY-BODY QUANTUM SYSTEMS}

In this section, we introduce the many-body Hamiltonians we use in this paper (Sec. II A) and then present a general overview of the coefficient distributions (Sec. IIB). The distributions are close to Gaussian away from the spectral edges in nonintegrable systems. They deviate significantly from Gaussianity for many eigenstates in integrable systems, and for eigenstates at the spectral edges in all systems. In Sec. IIC we quantify the deviation from Gaussianity using the Kullback-Leibler divergence and investigate the degree of Gaussianity in various cases using this measure. We show that, with increasing system size, resemblance to Gaussian form improves for nonintegrable systems but deteriorates for integrable systems.

\section{A. Models}

We consider the spin- $-\frac{1}{2} X X Z$ and Bose-Hubbard systems, on finite one-dimensional (1D) chains. We use open boundary conditions to avoid complications due to translation symmetry. For the $X X Z$ chain, a next-nearest-neighbor (NNN) coupling breaks integrability:

$$
H_{X X Z}=\sum_{i=1}^{L-1} h_{i, i+1}+\lambda \sum_{i=2}^{L-2} h_{i, i+2},
$$

where $h_{i, j}=S_{i}^{x} S_{j}^{x}+S_{i}^{y} S_{j}^{y}+\Delta S_{i}^{z} S_{j}^{z}$ (with spin- $\frac{1}{2}$ operators $S_{i}^{x, y, z}$ ) and $L$ is the number of sites. The NNN (second) term excludes the coupling between sites 1 and 3 , breaking reflection symmetry for $\lambda \neq 0$. We use $\Delta=0.8$ throughout this work.

The Bose-Hubbard chain is described by the Hamiltonian

$$
H_{\mathrm{BH}}=\sum_{i=1}^{L-1}\left(b_{i}^{\dagger} b_{i+1}+b_{i+1}^{\dagger} b_{i}\right)+\lambda \sum_{i=1}^{L} b_{i}^{\dagger} b_{i}^{\dagger} b_{i} b_{i},
$$

where $b_{i}$ denotes the bosonic annihilation operator on site $i$.

The number $N_{\uparrow}$ of "up" spins (XXZ) and the number of bosons $N_{\mathrm{b}}$ (Bose-Hubbard) are conserved quantities. We study a single sector at a time, i.e., we fix $\left(L, N_{\uparrow}\right)$ or $\left(L, N_{\mathrm{b}}\right)$. The Hilbert space dimensions are $D=\left(\begin{array}{c}L \\ N_{\uparrow}\end{array}\right)$ for the $X X Z$ chain and $D=\left(\begin{array}{c}L+N_{\mathrm{b}}-1 \\ N_{\mathrm{b}}\end{array}\right)$ for the Bose-Hubbard system.
In both Hamiltonians (1) and (2), the second term breaks integrability; the dimensionless parameter $\lambda$ controls proximity to integrability. The two integrable $(\lambda=0)$ Hamiltonians are the nearest-neighbor $X X Z$ Hamiltonian, which is integrable by Bethe ansatz, and a chain of free (noninteracting) bosons, which is integrable due to the absence of interactions. We will present data mostly for $\lambda=1$ (typical nonintegrable case) and $\lambda=0$ (integrable case).

In addition to the Hamiltonian classes (1) and (2), for our detailed treatment of integrable systems we will also consider a tight-binding system of $N_{\mathrm{f}}$ free fermions on an $L$-site chain,

$$
H_{\mathrm{FF}}=\sum_{i}\left(c_{i}^{\dagger} c_{i+1}+c_{i+1}^{\dagger} c_{i}\right)+\sum_{i} V_{i} c_{i}^{\dagger} c_{i},
$$

subject to a weakly varying potential $V_{i}$ which leaves the system integrable but avoids lattice symmetries. Here, $c_{i}$ denotes the fermionic annihilation operator on site $i$. The Hamiltonian conserves the fermion number $N_{\mathrm{f}}$. The Hilbert space dimension is $D=\left(\begin{array}{c}L \\ N_{\mathrm{f}}\end{array}\right)$.

\section{B. Gaussian and non-Gaussian distributions}

We are interested in the statistics of coefficients $c_{\gamma}^{(\alpha)} \equiv$ $\left\langle\phi_{\gamma} \mid \psi_{\alpha}\right\rangle$ of the energy eigenstates $\left|\psi_{\alpha}\right\rangle$. The basis states $\left\{\left|\phi_{\gamma}\right\rangle\right\}$ are spatial configurations, i.e., eigenstates of the local operators $S_{i}^{z}$ or $b_{i}^{\dagger} b_{i}$. Normalization ensures that $\sum_{\gamma=1}^{D}\left|c_{\gamma}^{(\alpha)}\right|^{2}=1$. We study distributions of $z=\left|c_{\gamma}\right| \sqrt{D}$ (eigenstate indices $\alpha$ are suppressed). These distributions $P(z)$ then have unit variance, which simplifies comparison between different sizes.

In Fig. 1 we show the distributions of coefficients of 250 eigenstates, taken from the edges and from the middle of the spectra. States at the edge are special; they tend to be nongeneric ("integrable-like"). In the coefficients this is manifested by non-Gaussian distributions, regardless of whether the system is integrable or not. For integrable many-body systems, e.g., the $X X Z$ chain with $\lambda=0$ [Fig. 1(b)] and other cases shown later, the distribution is also markedly non-Gaussian for eigenstates in the middle of the spectrum.

In contrast to the cases discussed above, for nonintegrable systems (e.g., the $X X Z$ chain with NNN coupling at $\lambda=1$ ), the distribution of the coefficients of the eigenstate in the middle of the spectrum has overall Gaussian behavior; see Fig. 1(a). The same is valid for the Bose-Hubbard chain with
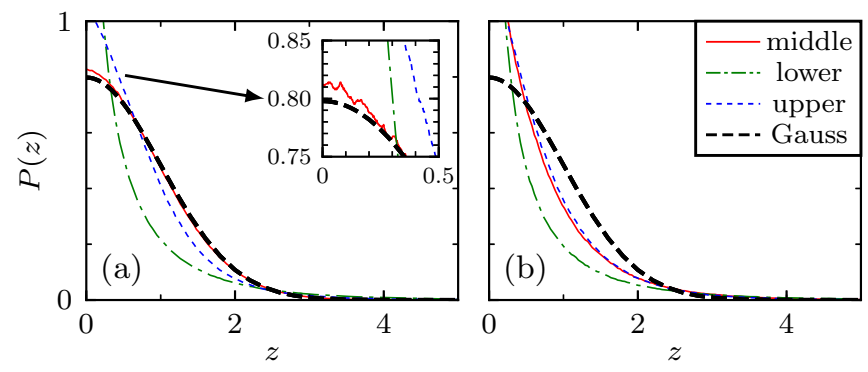

FIG. 1. (a) Amplitude distributions for nonintegrable $X X Z$ chain, with $\left(L, N_{\uparrow}\right)=(17,8), \Delta=0.8$, and $\lambda=1$. The distributions are over 250 eigenstates in the middle ( $E \approx 0$ ), at the lower edge, and at the upper edge of the spectrum. The black dashed curve is the Gaussian distribution with unit variance. The inset shows a magnification near zero. (b) The same, for the integrable $X X Z$ chain, with $\lambda=0$. 
$\lambda \sim 1$ (not shown). A Gaussian $P(z)$ is expected for complex nonintegrable Hamiltonians; it is equivalent to the PorterThomas distribution for $|z|^{2}$ in nuclear physics [35] and has been assumed or tested for condensed-matter Hamiltonians, e.g., in Refs. [36-39].

We observe a weak deviation from the Gaussian close to zero (Fig. 1, inset). The small excess weight near $z=0$ is balanced at intermediate values of $z$; the distribution is lower than the Gaussian at intermediate $z$ and then overshoots the Gaussian curve again at large $z$. The overall distribution thus has higher kurtosis than the Gaussian, about 3.17 for the data shown in Fig. 1(a). The deviation is characterized in some detail in the following subsection.

\section{Deviation from Gaussianity}

We will now present a quantitative analysis of deviation from "Gaussianity." For this purpose, we use a commonly used measure of the difference between two distributions, namely the Kullback-Leibler divergence (KLD) [40]. The KLD between $P(z)$ and the standard Gaussian distribution $P^{\mathrm{G}}(z)$ is

$$
D_{\mathrm{KL}}\left(P \| P^{\mathrm{G}}\right)=\int_{0}^{\infty} P(z) \ln \frac{P(z)}{P^{\mathrm{G}}(z)} d z .
$$

This quantity vanishes if $P(z)$ is identical to $P^{\mathrm{G}}(z)$ and grows as $P(z)$ increasingly deviates from $P^{\mathrm{G}}(z)$.

In Figs. 2(a) and 2(b), the KLD for each eigenstate is plotted against corresponding eigenenergies. Consistent with Fig. 1(a), in the nonintegrable case [Fig. 2(a)], the $D_{\mathrm{KL}}$ values are close to zero in the middle and larger at the edges of the spectrum. In the integrable case [Fig. 2(b)], there is a large spread of $D_{\mathrm{KL}}$ throughout the spectrum.

This behavior is reminiscent of that of bipartite entanglement entropy (EE) $S_{\alpha}$ of eigenstates [41]: in integrable systems, the middle of the spectrum has both generic, highEE eigenstates but also a substantial number of nongeneric, low-EE eigenstates [42,43], while nonintegrable systems have only high-EE eigenstates in the middle of the spectrum $[42,44,45]$. This suggests that the KLD of an eigenstate is inversely correlated with EE, and that large KLD and small EE both represent deviations from generic (effectively random or "thermal") behavior.

The correlation between the KLD and EE is shown in Figs. 2(c)-2(e), using scatter plots of the per-eigenstate KLD against the per-eigenstate exponentiated $\operatorname{EE}, \exp \left(S_{\alpha}\right)$. Here, the entanglement is between two spatially connected parts of the Bose-Hubbard chain, of sizes $l$ and $l+1$, where $2 l+1=L$. The data exhibit a very significant correlation between the KLD and EE, with improving correlation for increasing system size. We quantify this correlation using Pearson correlation coefficients $\rho$ between $\exp S_{\alpha}$ and $\ln D_{\mathrm{KL}}$, which measures how linear the correlation between these two quantities is. The coefficient is negative because larger-KLD states generally have smaller entanglement, i.e., the plots overall have negative slope. The magnitude of $\rho$ increases steadily with system size. Similar behavior is observed for the $X X Z$ model with NNN couplings (not shown), which suggests that the improvement of correlation with increasing system size is a generic feature.

The participation ratio (PR) of eigenstates in the real-space configuration basis is more directly correlated with the KLD.
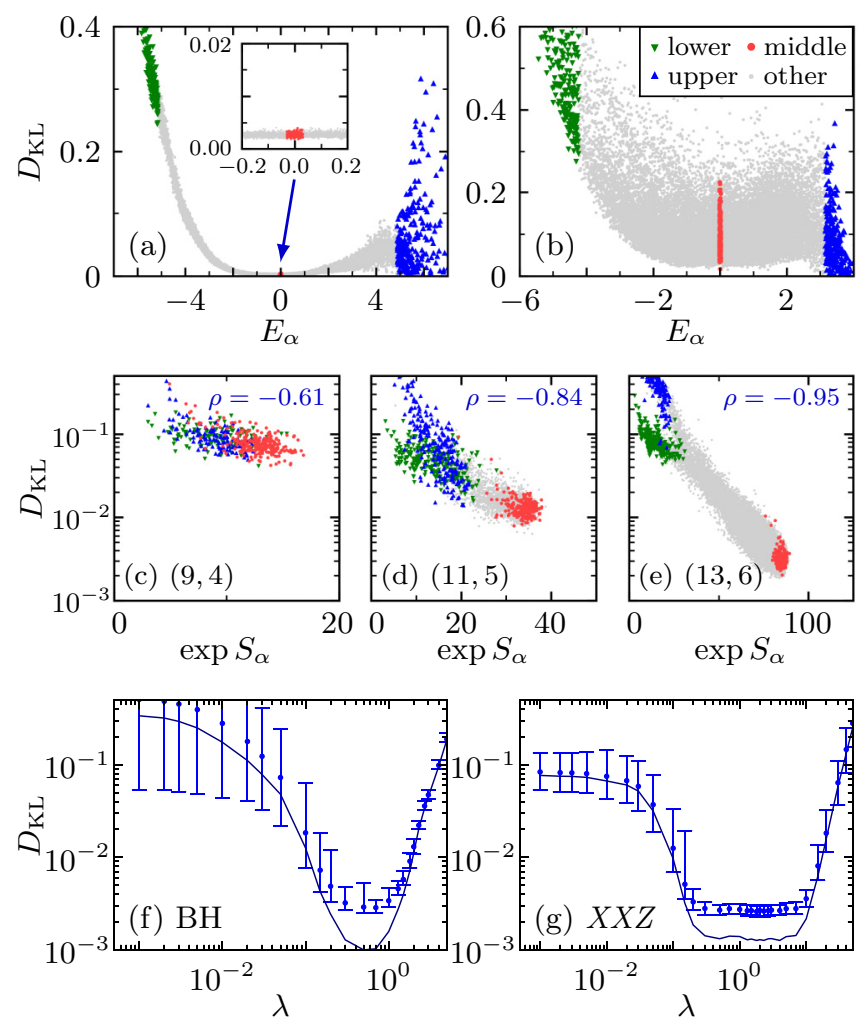

FIG. 2. Kullback-Leibler divergence $D_{\mathrm{KL}}$ (deviation from Gaussianity) for $X X Z$ and for Bose-Hubbard. (a) Per-eigenstate $D_{\mathrm{KL}}$ versus eigenenergy $E_{\alpha}$ for the nonintegrable $X X Z$ chain with NN coupling $\left[\left(L, N_{\uparrow}\right)=(17,8), \Delta=0.8, \lambda=1\right]$. (b) The same for the integrable $X X Z$ chain (same size, $\lambda=0$ ). The highlighted states correspond to distributions shown in Fig. 1. (c-e) Per-eigenstate $D_{\mathrm{KL}}$ against exponentiated entanglement entropy for three system sizes $\left(L, N_{\mathrm{b}}\right)=(9,4),(11,5),(13,6)$, respectively, of the Bose-Hubbard model $(\lambda=1)$. We indicate the Pearson correlation coefficients $\rho$. (f) $D_{\mathrm{KL}}$ as function of $\lambda$ for the Bose-Hubbard chain $\left[\left(L, N_{\mathrm{b}}\right)=(13,6)\right]$. Data points and error bars are average and standard deviation of the per-eigenstate $D_{\mathrm{KL}}$ values for 250 eigenstates in the middle of the spectrum. Solid line is $D_{\mathrm{KL}}$ of the distribution of all coefficients of these states together. $(\mathrm{g})$ The same for the $X X Z$ chain with $\mathrm{NNN}$ coupling $\left[\left(L, N_{\uparrow}\right)=(17,8), \Delta=0.8\right]$.

The inverse PR

$$
p^{-1}=D \sum_{\gamma}\left|c_{\gamma}\right|^{4}=\int z^{4} P(z) d z
$$

is the kurtosis of the coefficient distribution, having the value $p=1 / 3$ for a Gaussian distribution. The (inverse) PR has been used as a characterization of proximity to integrability $[5,28,42,46]$.

Like the KLD, the PR can be calculated directly from the shape of the coefficient distribution; in contrast, the EE involves a partial trace which requires additional information about the spatial structure of the basis states. In view of the correlation between EE and KLD displayed in Figs. 2(c)-2(e), it is thus expected that the EE and the PR should be positively correlated, as explored in Ref. [42]. We provide some further data in Appendix B. 

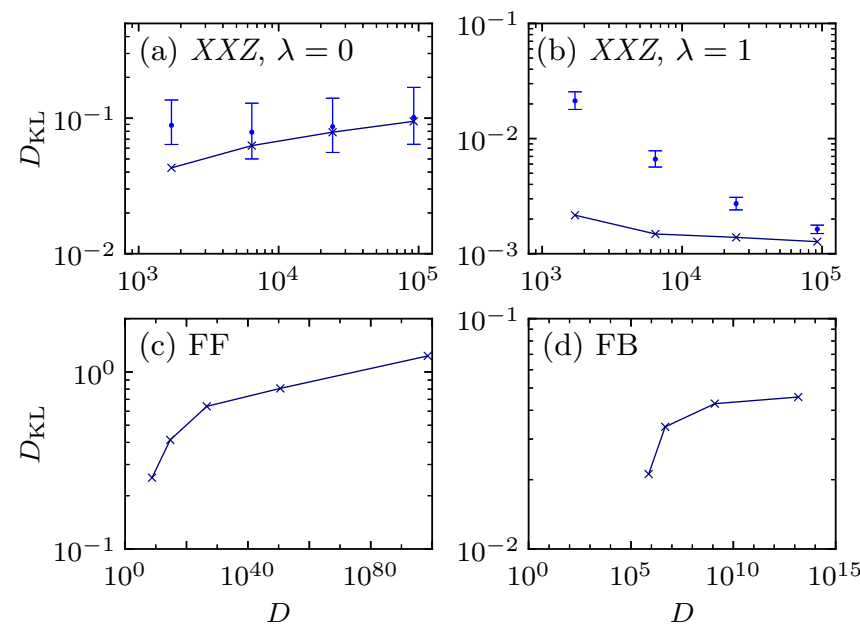

FIG. 3. Kullback-Leibler divergence $D_{\mathrm{KL}}$ as function of the system size (Hilbert space dimension $D$ ). (a) The integrable $X X Z$ chain ( $\Delta=0.8, \lambda=0$ ) for 250 eigenstates in the middle of the spectrum. The data points (dots) and error bars indicate the average and standard deviation of the per-state KLD. The solid line (crosses) shows the $D_{\mathrm{KL}}$ values of the distribution of the coefficients of the 250 states taken together. (b) The same for the nonintegrable $X X Z$ chain with NNN couplings $(\lambda=1)$. (c) Eigenstate-averaged KLD for free fermions (FF). (d) Eigenstate-averaged KLD for free bosons (FB).

Figures 2(f) and 2(g) show the KLD as a function of the integrability-breaking parameter $\lambda$. In the nonintegrable regime $(\lambda \sim 1)$ the coefficient distribution for every eigenstate in the middle of the spectrum is close to Gaussian, with $D_{\mathrm{KL}}$ near zero. For $\lambda \rightarrow 0$, the values of $D_{\mathrm{KL}}$ grow, and there is a large variation between the different eigenstates, reflecting the large spread of $D_{\mathrm{KL}}$ values in Fig. 2(b). For $\lambda \gg 1, D_{\mathrm{KL}}$ increases rapidly. In this limit, local conserved quantities divide the Hilbert space into uncoupled sectors, leading to a large number of zero coefficients, which accounts for the strong deviation from Gaussianity.

Figure 3 shows the KLD as a function of system size. The smallest and largest system sizes for the $X X Z$ chain [Figs. 3(a) and 3(b)] correspond to $L=13$ and 19. For the free-fermion and free-boson chains [Figs. 3(c) and 3(d)], the accessible sizes are much larger, because the coefficient distributions can be obtained without explicit numerical diagonalization of the many-body Hamiltonians, using the fact that each many-body eigenstate is built out of single-particle eigenstates as a single Slater determinant (noninteracting fermions) or as a single permanent (noninteracting bosons). The issue is discussed further in Sec. III A.

In the nonintegrable $X X Z$ chain [Fig. 3(b)], there is a clear decrease of $D_{\mathrm{KL}}$ (increasing Gaussianity) with increasing system size. For the integrable cases, both the integrable $X X Z$ chain and the noninteracting systems, $D_{\mathrm{KL}}$ increases with system size, meaning that $P(z)$ becomes less Gaussian. This is consistent with our conjecture in the next section that $P(z)$ approaches a power law in the large-size limit. In addition, relatively large fluctuations between the eigenstates are observed [error bars in Fig. 3(a)], reflecting the broad distribution seen in Fig. 1(b). This is consistent with the idea that eigenstates of integrable systems have a nonuniversal structure at finite sizes.

\section{INTEGRABLE MANY-BODY SYSTEMS}

We now concentrate on integrable systems and consider the coefficient distribution in the limit of large sizes. First, we describe the numerical analysis that leads to the conjecture of power-law behavior at large sizes (Sec. III A). The rest of the section provides a series of analytical arguments in support of this conjecture.

\section{A. Numerical analysis}

The eigenstate coefficients for free bosons and free fermions can be evaluated without explicit diagonalization of the many-body Hamiltonian, using the fact that the many-body eigenstates are built out of single-particle eigenstates. The eigenstates are chosen by drawing the "momenta" $k_{j}$ randomly such that the many-body energies lie in the desired energy range. For larger Hilbert spaces, we typically sample $10^{3}-10^{4}$ coefficients of each eigenstate. For free fermions, the eigenfunctions are (Slater) determinants, which can be evaluated efficiently, allowing us to sample relatively large systems ( $>300$ sites). For the $X X Z$ chain, we are limited to exact diagonalization and the sizes are modest $(\approx 20$ sites $)$. Intermediate are free bosons ( $>30$ sites), whose eigenfunctions are permanents, whose numerical evaluation is less favorable than determinants.

Figures 4(a)-4(c) present double-logarithmic plots of the coefficient distribution for three integrable systems. A powerlaw behavior would show up as a straight line in this representation. The data in the free fermionic case show a clear evolution toward power-law behavior as the system size is increased. The trend in the other two systems is in the same direction, but less pronounced, presumably because of limited system sizes. The data are further analyzed in Figs. 4(d)-4(f) through the slope of the curve in the double-logarithmic plot,

$$
\frac{d \ln P}{d \ln z}=\frac{z}{P} P^{\prime}(z),
$$

the double-logarithmic derivative. Power-law behavior of $P$ would imply a constant (flat) double-logarithmic derivative, its value giving the power-law exponent. The inset shows that the slope of the double-logarithmic derivative in logarithmic scale,

$$
k=\frac{d^{2} \ln P}{d(\ln z)^{2}},
$$

becomes smaller, arguably scaling to zero, in the large-size limit $N_{\mathrm{f}} \rightarrow \infty$. The available sizes do not allow a meaningful extrapolation for the other two systems, but show the same general trend. This observation, together with further supporting arguments below, lead to the conjecture that the tails of the coefficient distributions of eigenfunctions of integrable manybody systems approach power-law shapes in the large-size limit.

\section{B. Structure of the many-body coefficients}

A common feature of several types of integrable systems is that many-body eigenstates can be constructed out of singleparticle eigenstates. For example, a two-particle wave function is of the form $\phi_{a}(1) \phi_{b}(2) \mp \phi_{a}(2) \phi_{b}(1)$ for fermions and 

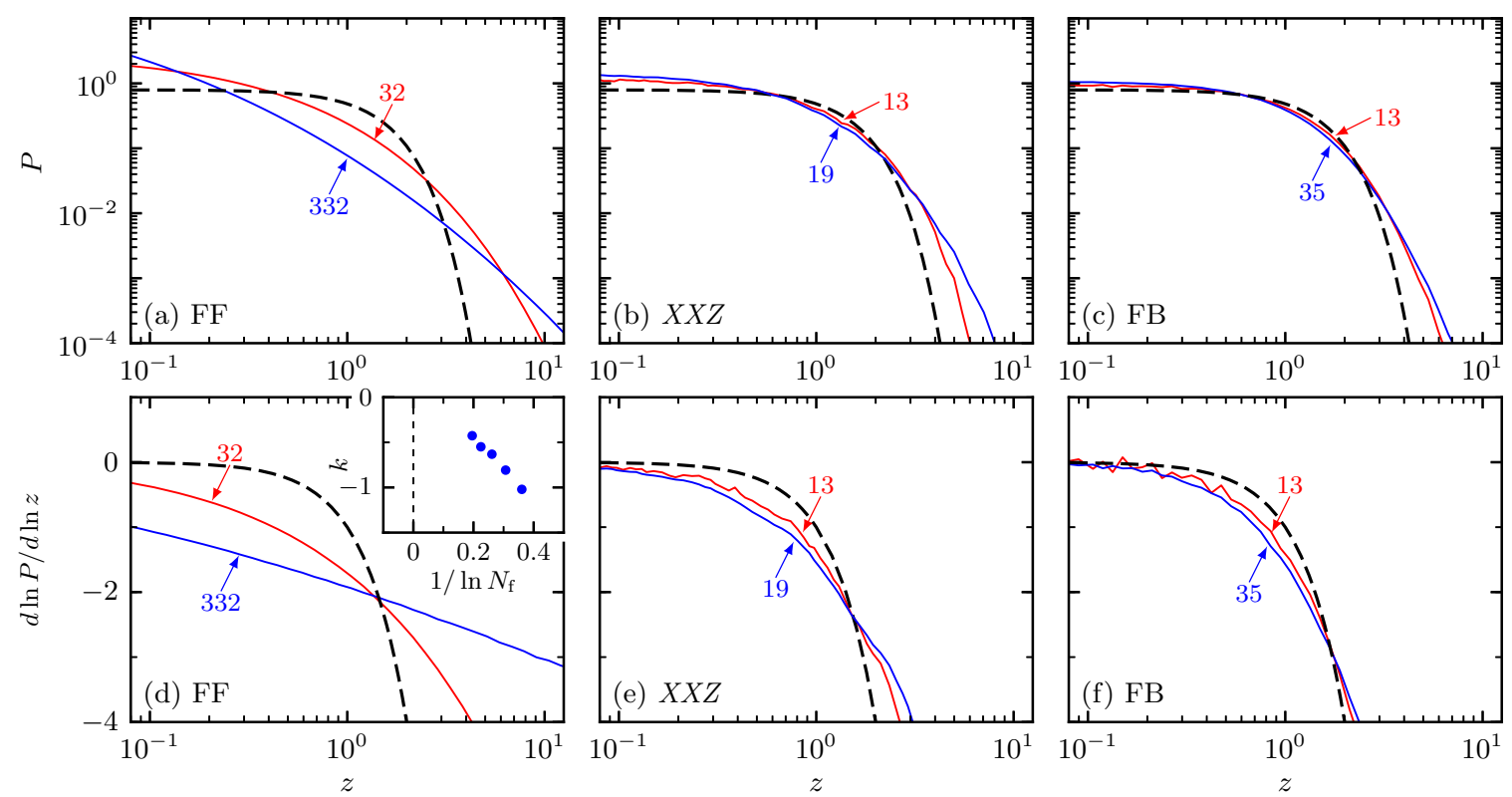

FIG. 4. (a-c) Amplitude distributions for integrable many-body systems, sampled from multiple eigenstates in the middle of the spectrum. Dashed curves are Gaussians. (a) Free-fermion (FF) chain; $\left(L, N_{\mathrm{f}}\right)=(32,16)$ and $(332,166),\left(D \sim 10^{8}\right.$ and $\left.10^{98}\right)$. (b) $X X Z$ chain at $\Delta=0.8$, with $\left(L, N_{\uparrow}\right)=(13,6)$ and $(19,9)\left(D=1716\right.$ and 92378). (c) Free-boson (FB) chain; $\left(L, N_{\mathrm{b}}\right)=(13,6)$ and $(35,17)\left(D \sim 10^{4}\right.$ and $\left.10^{13}\right)$. (d-f) Corresponding double-logarithmic derivatives. Inset in (d) is the "curvature" $k=\frac{d^{2} \ln P}{d(\ln z)^{2}}$ as function of $1 / \ln N_{\mathrm{f}}$ for the free-fermion chain.

bosons and of the form $\phi_{a}(1) \phi_{b}(2)+e^{i \chi} \phi_{a}(2) \phi_{b}(1)$ for the $X X Z$ chain, where $\chi$ is a phase shift and $\phi_{a, b}$ are single-particle eigenstates. We now consider the structure of the many-body coefficients for larger system sizes, as to eventually obtain information about their distribution.

Let us consider the single-particle Hamiltonian corresponding to the integrable many-body system of interest. This contains hopping terms and possibly a background potential,

$$
H=\sum_{i=1}^{L-1}\left(a_{i}^{\dagger} a_{i+1}+a_{i+1}^{\dagger} a_{i}\right)+\sum_{i=1}^{L} V_{i} a_{i}^{\dagger} a_{i} .
$$

(The geometry could be something other than a chain, e.g., a two-dimensional (2D) or three-dimensional (3D) lattice, and the hoppings could be longer-range, without affecting any of the arguments below.) The creation and annihilation operators above can have any exchange statistics, for example, but not limited to, fermionic or bosonic.

The creation operators for the single-particle eigenstates are linear combinations of the $a^{\dagger}$ operators,

$$
d_{k}^{\dagger}=\sum_{j=1}^{L} \phi_{j}^{(k)} a_{j}^{\dagger}
$$

Here, $k=1, \ldots, L$ labels the single-particle eigenstates, and the $j$ are site indices. The $\phi_{j}^{(k)}$ are single-particle eigenstate coefficients. For integrable systems, the many-body eigenstate coefficients are built out of these $\phi_{j}^{(k)}$.

In the simplest situation of nearest-neighbor hopping with no background potential, the $\phi_{j}^{(k)}$ are sine functions, e.g., with open boundary conditions,

$$
\phi_{j}^{(k)}=\sqrt{\frac{2}{L+1}} \sin \frac{k j \pi}{L+1} .
$$

In this case, the indices $k$ can be interpreted as momenta. The corresponding single-particle energies are $E^{(k)}=2 \cos \frac{k \pi}{L+1}$. The arguments below do not rely on a specific form of the single-particle eigenstates and energies.

For noninteracting bosons or fermions, the many-body eigenstates are constructed by filling the single-particle eigenstates with integer numbers of particles. The eigenstates can be labeled either as a list of occupancies of the $L$ single-particle eigenstates,

$$
\left|\tilde{n}_{1}, \ldots, \tilde{n}_{L}\right\rangle=\prod_{k=1}^{L} \frac{1}{\tilde{n}_{k} !}\left(d_{k}^{\dagger}\right)^{\tilde{n}_{k}}|\mathrm{vac}\rangle
$$

or as a list of the single-particle eigenstates occupied by the $N$ particles,

$$
\left|k_{1}, \ldots, k_{N}\right\rangle=d_{k_{1}}^{\dagger} \cdots d_{k_{N}}^{\dagger}|\mathrm{vac}\rangle
$$

Here, $|\mathrm{vac}\rangle$ is the vacuum (no particles in the system). The integers $\tilde{n}_{k} \geqslant 0$ indicate how many particles are in singleparticle eigenstate $|k\rangle$. For fermions, $\tilde{n}_{k}=0,1$ and for bosons they can take values up to $N$. The many-body eigenenergy is equal to $\sum_{k=1}^{L} \tilde{n}_{k} E^{(\mathrm{k})}$.

For noninteracting bosons, the eigenstates can be expressed as the sum over permutations $p$ of the positions $\left(j_{1}, \ldots, j_{N}\right)$ of the $N$ particles. In the basis defined by the states $\left|j_{1}, \ldots, j_{N}\right\rangle \equiv$ $a_{j_{1}}^{\dagger} \cdots a_{j_{N}}^{\dagger}|\mathrm{vac}\rangle$, the eigenstate coefficients are

$$
\left\langle j_{1}, \ldots, j_{N} \mid k_{1}, \ldots, k_{N}\right\rangle=\frac{\sqrt{\gamma_{\{j\}}}}{\sqrt{\tilde{\gamma}_{\{k\}}}} \sum_{p \in \mathcal{P}} \phi_{p_{1}}^{\left(k_{1}\right)} \phi_{p_{2}}^{\left(k_{2}\right)} \cdots \phi_{p_{n}}^{\left(k_{n}\right)},
$$

where $\tilde{\gamma}_{\{k\}}=\prod_{k=1}^{L} \tilde{n}_{k} ! \quad$ and $\quad \gamma_{\{j\}}=\prod_{j=1}^{L} n_{j} !$, and $p=$ $\left(p_{1}, \ldots, p_{N}\right)$ runs over all distinct permutations of the particle 
positions $\left(j_{1}, \ldots, j_{N}\right)$. The summation may be conveniently implemented as the permanent per $M$ of the $N \times N$ matrix $M$ defined by $M_{a b}=\phi_{j_{a}}^{\left(k_{b}\right)}(a, b=1, \ldots, N)$.

For free fermions, the many-body eigenstates are linear combinations of the products of the single-particle eigenstates, as for free bosons. However, antisymmetry under exchange of particles introduces minus signs in this sum for odd permutations. The coefficients are therefore given by Slater determinants

$$
\begin{aligned}
& \left\langle j_{1}, \ldots, j_{N} \mid k_{1}, \ldots, k_{N}\right\rangle \\
& =\frac{1}{\sqrt{N !}} \operatorname{det} M=\frac{1}{\sqrt{N !}} \sum_{p \in \mathcal{P}}(-1)^{p} \phi_{p_{1}}^{\left(k_{1}\right)} \phi_{p_{2}}^{\left(k_{2}\right)} \cdots \phi_{p_{n}}^{\left(k_{n}\right)} .
\end{aligned}
$$

The single-particle eigenstates $k_{i}$ are required to be distinct.

For systems solvable by the Bethe ansatz, the many-body amplitudes are also built out of single-particle coefficients. For the fermionic chain with nearest neighbor interactions (equivalent to the $X X Z$ chain for present purposes),

$$
\left\langle j_{1}, \ldots, j_{N} \mid k_{1}, \ldots, k_{N}\right\rangle=\mathcal{N} \sum_{p \in \mathcal{P}} \mathrm{e}^{\mathrm{i} \chi(p)} \phi_{p_{1}}^{\left(k_{1}\right)} \phi_{p_{2}}^{\left(k_{2}\right)} \cdots \phi_{p_{n}}^{\left(k_{n}\right)},
$$

where the phase shift $\chi(p)=\sum_{i<j} \chi_{2}\left(k_{i}, k_{j}\right)$ is interaction dependent and is a sum of two-particle phase shifts $\chi_{2}$, which are determined from the two-particle scattering problem. For more complicated models, such as those requiring the nested Bethe ansatz, the wave function is more involved, but the essential idea is the same.

\section{Argument for power-law behavior: A toy model}

We provide an argument for power-law behavior of the coefficient distribution by considering a toy model of $N$ distinguishable particles, i.e., with trivial exchange statistics. In this case, the multiparticle eigenfunction coefficients

$$
c=\left\langle j_{1}, \ldots, j_{N} \mid k_{1}, \ldots, k_{N}\right\rangle=\prod_{i=1}^{N}\left\langle j_{i} \mid k_{i}\right\rangle=\prod_{i=1}^{N} \phi_{j_{i}}^{\left(k_{i}\right)}
$$

are merely products of values of the single-particle eigenstates $\phi_{j}^{(k)}$, where $k$ label the eigenstates and $j$ the site indices; $c$. Eqs. (13)-(15). For $N$ distinguishable particles in $L$ sites, the Hilbert space dimension is $D=L^{N}$.

Because of the product-state nature of the coefficients $c$, it is natural to study the distributions $Q(y)$ of the logarithms

$$
y=\ln z=\ln c+\ln \sqrt{D} .
$$

The distribution $Q(y)$ relates to the "usual" coefficient distribution $P(z)$ as $Q(y)=\mathrm{e}^{y} P\left(\mathrm{e}^{y}\right)$ and $P(z)=\frac{1}{z} Q(\ln z)$. The logarithm of the many-particle coefficients is the sum of the single-particle ones, $\ln c=\sum_{i=1}^{N} \ln \phi_{j_{i}}^{\left(k_{i}\right)}$, so that

$$
y=\sum_{i=1}^{N} \ln \phi_{j_{i}}^{\left(k_{i}\right)}+\frac{1}{2} N \ln L .
$$

If we regard the single-particle coefficients to be effectively random, then this is a sum of $N$ random variables (plus a shift by a constant), and we can invoke the central limit theorem. It follows that $Q(y)$ at large $N$ approaches a Gaussian distribution,

$$
Q(y) \rightarrow \frac{1}{\sqrt{2 \pi \sigma_{Q}^{2}}} \mathrm{e}^{-\left(y-\mu_{Q}\right)^{2} / 2 \sigma_{Q}^{2}},
$$

with mean $\mu_{Q}$ and variance $\sigma_{Q}^{2}$. The central limit theorem yields the mean to be the sum $\mu_{Q}=\sum_{i=1}^{N} \mu_{q_{i}}$ of the means $\mu_{q_{i}}$ of the single-particle log-coefficient distributions $q_{i}$ of the variables $y_{i}=\ln \phi_{j}^{\left(k_{i}\right)}+\frac{1}{2} \ln L$ [i.e., one term in the sum of Eq. (18)]. The term $\frac{1}{2} \ln L$ represents the scaling of the single-particle coefficients to unit variance, which renders the distributions $q_{i}$ to be independent of system size in the limit $L \rightarrow \infty$. The values $\mu_{q_{i}}$ only depend on the lattice geometry and the quadratic couplings (e.g., short-range versus long-range couplings). Likewise, the variance $\sigma_{Q}^{2}$ approaches $\sum_{i=1}^{N} \sigma_{q_{i}}^{2}$ for large $N$, where the $\sigma_{q_{i}}^{2}$ are the variances of the single-particle distributions $q_{i}$.

The power-law behavior of $P(z)=\frac{1}{z} Q(\ln z)$ now readily follows from studying the first- and second-order doublelogarithmic derivative,

$$
\begin{gathered}
\frac{d \ln P}{d \ln z}=-\frac{\ln z}{\sigma_{Q}^{2}}+\frac{\mu_{Q}}{\sigma_{Q}^{2}}-1, \\
k=\frac{d^{2} \ln P}{d(\ln z)^{2}}=-\frac{1}{\sigma_{Q}^{2}} .
\end{gathered}
$$

Under the assumption of identical distributions $q_{i}=q$, we have $\sigma_{Q}^{2}=N \sigma_{q}^{2}$ and $\mu_{Q}=N \mu_{q}$ and find that the secondorder double-logarithmic derivative scales as $k \sim 1 / N$. Thus, for increasing system size, the curvature of the coefficient distribution $P(z)$ on a double logarithmic scale decreases. In other words, $P(z)$ "flattens" to a power law $\sim z^{\alpha}$. The exponent $\alpha$ of the power law follows from the first-order double-logarithmic derivative, $\alpha \rightarrow \mu_{q} / \sigma_{q}^{2}-1$ (at $z=1$ ). The value is nonuniversal: it is determined by the details of the single-particle coefficient distributions.

In Fig. 5 we illustrate the effectiveness of this argument with the results for a model of many distinguishable particles in a finite chain with open boundary conditions (many particles in a box). The numerically obtained distribution fits well to the analytic estimate given by Eq. (19) with $\mu_{Q}=N \mu_{q}$ and $\sigma_{Q}^{2}=$ $N \sigma_{q}^{2}$. (In this case, the single-particle coefficient distributions are independent of the $k_{i}$; they are all characterized by the same mean $\mu_{q}$ and variance $\sigma_{q}^{2}$. We find $\mu_{q} \approx-0.347$ and $\sigma_{q}^{2} \approx 0.822$, which yields the exponent $\alpha \approx-1.42$.) Small deviations may be seen in the tails, and the numerical distribution is slightly skewed to the right, due to the high asymmetry of the single-particle distribution $q$. These deviations vanish in the limit $N \rightarrow \infty$.

\section{Extension to nontrivial statistics}

From the data in Fig. 4, the question arises as of whether the preceding argumentation for a large- $N$ approach to a power-law $P(z)$ can be extended naturally to indistinguishable particles with nontrivial statistics. For free bosons, free fermions and systems integrable through the Bethe ansatz, 

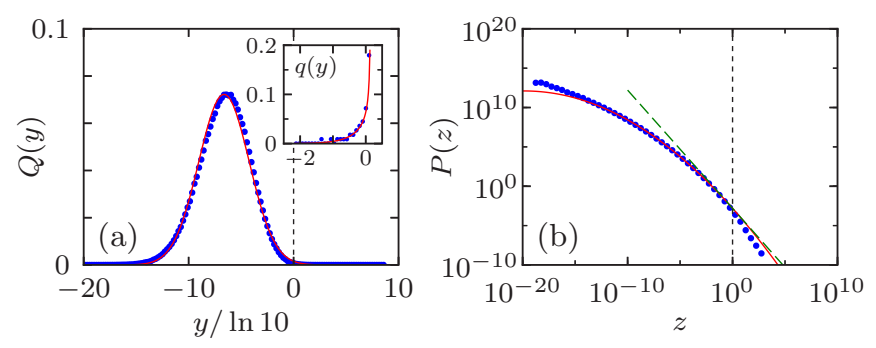

FIG. 5. (a) Distribution $Q(y)$ of the logarithms of the coefficients of the many-particle-in-a-box model, for $(L, N)=(100,50)$. We consider a single eigenstate, with randomly chosen momenta $\left(k_{1}, \ldots, k_{N}\right)$. The (blue) dots indicate the numerical result obtained by sampling $10^{6}$ coefficients. The (red) curve is the estimated distribution $Q$ from Eq. (19), with mean $\mu_{Q}$ and variance $\sigma_{Q}^{2}$ obtained from the single-particle distributions. The inset shows a typical single-particle distribution $q(y)$, with the dots and curve indicating the discrete and continuum distributions, respectively. (b) Corresponding coefficient distribution $P(z)$. The (green) dashed line is an estimate for the power law with exponent evaluated at $z=1$.

the eigenfunctions are not just products of single-particle wave functions, but linear combinations of them, as shown in Eqs. (13)-(15), respectively. At present, we are able to outline a partial argument only for the free-fermion case.

For free fermions, the many-body coefficients are determinants of the single-particle coefficients, Eq. (14). Assuming these coefficients to be effectively random, we invoke recent results from random matrix theory for the determinant of a random matrix $[47,48]$. Assuming that the entries of a matrix $A$ are essentially random, and their distribution is sufficiently well-behaved, $\ln |\operatorname{det} A|$ satisfies a central-limit theorem: If the entries are distributed with zero mean and unit variance, the distribution of $\ln |\operatorname{det} A|$ tends to a normal distribution for large $N$, with mean $\frac{1}{2} \ln (N-1)$ ! and variance $\frac{1}{2} \ln N$. For our matrix $M$ in Eq. (14), the entries are single-particle coefficients $\phi_{j}^{(k)}$ with variance $1 / L$ by normalization. The matrix elements can be made to have unit variance by multiplying each element by $\sqrt{L}$, so that the determinant is multiplied by $(\sqrt{L})^{N}$. Thus, the coefficients are of the form

$$
c=\frac{1}{\sqrt{N !}} \frac{1}{L^{N / 2}} \operatorname{det} \tilde{M},
$$

where the matrix $\tilde{M}$ now has entries with unit variance. Unfortunately, the entries do not necessarily have zero average; for example, if the coefficients are sinusoidal functions as in the case of an open-boundary chain, half of the singleparticle coefficients have nonzero average. We proceed with the assumption that this nonzero average causes a shift $\xi$ in the mean of the distribution of $\ln |\operatorname{det} \tilde{M}|$, and leaves the variance unchanged. With this assumption, the variable $y=\ln (c \sqrt{D})$ has a Gaussian distribution $Q(y)$, as in Eq. (19), with mean

$$
\mu_{Q}=-\ln \sqrt{N !}-\ln L^{N / 2}+\frac{1}{2} \ln (N-1) !+\xi(N)+\ln \sqrt{D}
$$

and variance $\sigma_{Q}^{2}=\frac{1}{2} \ln N$
Gaussianity of $Q(y)$ implies Eqs. (20) and (21) for the firstand second-order double-logarithmic derivative. The latter

$$
k=-\frac{1}{\sigma_{Q}^{2}}=\frac{1}{\frac{1}{2} \ln N}
$$

thus vanishes at large $N$. This signifies an approach to powerlaw form for $P(z)$ in the $N \rightarrow \infty$ limit. Compared to the product-type states, the convergence is slower: $k \sim-1 / \ln N_{\mathrm{f}}$ [48]. This provides an appealing explanation to why we need enormous sizes to see the approach to power-law behavior and is the reason we plot $k$ against $1 / \ln N_{\mathrm{f}}$ in Fig. 4(d), inset.

We now attempt to estimate the power-law exponent at large sizes. Equation (20) implies that the exponent is

$$
\begin{aligned}
\frac{\mu_{Q}}{\sigma_{Q}^{2}}-1 & =\frac{-\frac{1}{2} \ln N-\ln L^{N / 2}+\ln \sqrt{D}+\xi(N)}{\frac{1}{2} \ln N}-1 \\
& =-2+\frac{-\ln L^{N / 2}+\ln \sqrt{D}+\xi(N)}{\frac{1}{2} \ln N}
\end{aligned}
$$

The fraction (second term) is $N$-dependent. [For the case of half-filling, $L=2 N$, the numerator is $-\frac{1}{2} N \ln N+\xi(N)$ at large $N$.] For a sensible large- $N$ limit, the $N$ dependence must be canceled by the unknown shift $\xi(N)$. If the cancellation is perfect in the sense that the fraction vanishes, we obtain the estimate -2 for the exponent, i.e., the asymptotic power-law behavior $P(z) \propto z^{-2}$, which is consistent with the numerical data presented in Fig. 4. Of course, since we do not know the function $\xi(N)$, the fraction could also be an $N$-independent constant, in which case the exponent would be shifted from -2 .

The assumption that the nonzero average of the matrix elements leads only to a shift in the mean of $Q(y)$ seems quite reasonable. Proving such an assumption, or deriving $\xi(N)$, is well beyond the scope of the present work. The central limit theorem for log-determinants, invoked above, is cutting-edge mathematical work. We are not aware of mathematical results with modified conditions for the distribution of elements. Note, however, that a power-law dependence can be inferred under much weaker conditions than the assumption used above; as long as $\sigma_{Q}^{2}$ is an increasing function of $N$, we obtain a power-law $P(z)$ at large $N$.

At present, to our knowledge, no comparable centrallimit-theorem analog is available for permanents [Eq. (13)] and certainly not for more complicated generalizations like Eq. (15) appearing in Bethe-ansatz wave functions, but a similar Gaussian limit for $Q(y)$, and hence a power-law $P(z)$ for large $N$, seems plausible. Thus, based on our numerical results and on the arguments above, a reasonable conjecture is that $P(z)$ approaches a power law generically in large-size integrable systems.

Any of these arguments (whether for trivial or for nontrivial statistics) rely on treating the single-particle logarithmic coefficients $\ln \phi_{a}$ as independent random variables. While such an assumption is likely impossible to be "proved," arguments in the same spirit underlie the eigenstate thermalization hypothesis (ETH) and its extensions [1-5,37,42,49-51]. 


\section{QUANTUM BILLIARDS}

In this section, we consider single-particle systems ("billiards") confined to a 2D region either by a hard wall (Sec. IV A) or by a parabolic confining potential (Sec. IV B). We show that a number of integrable billiards have amplitude distributions with power-law tails and present some data for systems with a mixed phase space.

\section{A. Hard walls}

We now consider a single particle confined in a $2 \mathrm{D}$ region $\Omega$. The eigenfunctions $\psi_{n}(\vec{x}), \vec{x}=(x, y)$, satisfy the Schrödinger equation $-\nabla^{2} \psi_{n}(\vec{x})=E_{n} \psi_{n}(\vec{x})$ for $\vec{x} \in \Omega$, and vanish for $\vec{x} \notin \Omega$. Given an eigenstate $\psi(\vec{x})$ of a quantum billiard, we consider the probability distribution $P(z)$ of the (rescaled) absolute values $z$ of the amplitudes, $z=|\psi(\vec{x})| \sqrt{\mathcal{A}}$,

$$
P(z) d z=\frac{1}{\mathcal{A}} \int_{z \leqslant|\psi(x, y)| \sqrt{\mathcal{A}}<z+d z} 1 d x d y .
$$

Here, $\mathcal{A}=\operatorname{area}(\Omega)$ is the area allowed by the billiard potential. Inclusion of the factor $\sqrt{\mathcal{A}}$ ensures that $P(z)$ has unit variance.

In contrast to chaotic quantum billiards, for which almost all eigenstates have Gaussian amplitude distributions [6-12,14], we here investigate integrable billiards, as in Ref. [52]. The simplest case is the square billiard, whose eigenfunctions $\psi_{n_{x} n_{y}}^{\square} \propto \sin \left(n_{x} x\right) \sin \left(n_{y} y\right)$ all have the same amplitude distribution, which can be expressed analytically in terms of an elliptic integral (see Appendix C) and is shown in Fig. 6(a). The tail of the distribution, which originates from the peaks of the wave function, is $\sim z^{-1 / 2}$, but there is no extended power-law
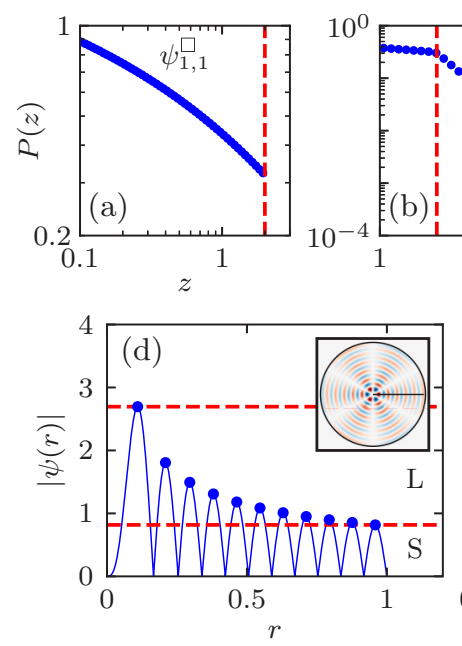
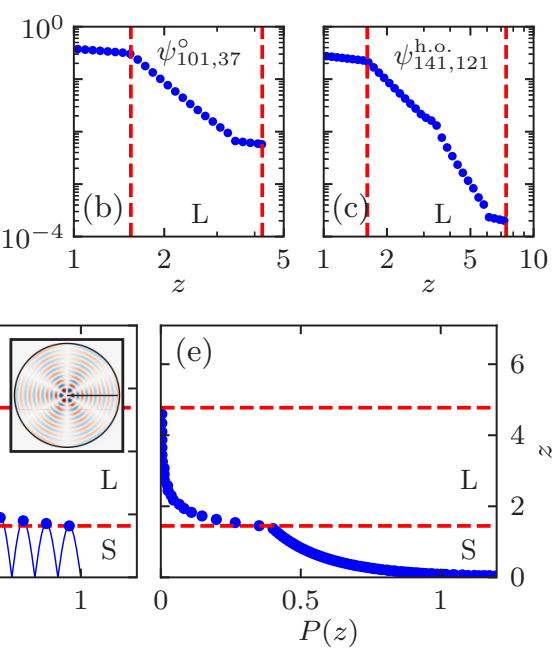

FIG. 6. Amplitude distributions in single-particle systems (loglog plots). Distributions shown for a single eigenstate of (a) The square billiard, $\psi_{1,1}^{\square}$. (b) The circular billiard, $\psi_{101,37}^{\circ}$. (c) An anisotropic 2D harmonic oscillator, $\psi_{141.121}^{\text {h.o. }}$ (d, e) Illustration of the origin of two distinct regimes (labeled S and L) of the amplitude distribution; here, for the circular billiard, eigenfunction $\psi_{3,11}^{\circ}$. In (d), the curve indicates $|\psi(r)|$ and the dots indicates local extrema. The inset visualizes $\psi_{3,11}^{\circ}(x, y)$. In (e), the resulting coefficient distribution is plotted sideways as function of $z$, which is scaled as to match the values of $|\psi(r)|$ in (d). In all panels, the (red) dashed lines indicate the "L" regime, where power-law behavior can be expected. (For the square billiard, this regime is undefined.) region. The circular billiard eigenstates $\psi_{m n}^{\circ}$ are labeled by angular and radial quantum numbers $m$ and $n$. At large $n$, the wave function has many oscillations in the radial direction, given by a Bessel function. This leads to a broad power-law segment in the amplitude distribution [see Fig. 6(b)]: $P(z) \sim$ $z^{-\gamma}$, with $\gamma \approx 5$ for $n \gg 1$ (see Appendix C). The region extends from the height of the lowest peak to that of the highest peak, as illustrated by Figs. 6(d) and 6(e). In Fig. 6 the expected power-law regime, defined by the minimum and maximum peak amplitude, is indicated by the (red) dashed lines.

\section{B. Soft walls}

These results also extend to single-particle eigenstates of smooth confining potentials, i.e., with Hamiltonian $H=$ $-\nabla^{2}+V(x, y)$. In this case, we need to restrict the analysis of the distribution to the classically accessible region, and define $\mathcal{A}$ [cf. Eq. (26)] to be the area of this region. Let us consider the $2 \mathrm{D}$ harmonic oscillator, $V_{\text {h.o. }}(x, y)=x^{2}+\lambda^{2} y^{2}$. The constant $\lambda=\frac{1}{2}(1+\sqrt{5})$ is taken to be irrational in order to avoid complications with degeneracies. The eigenfunctions
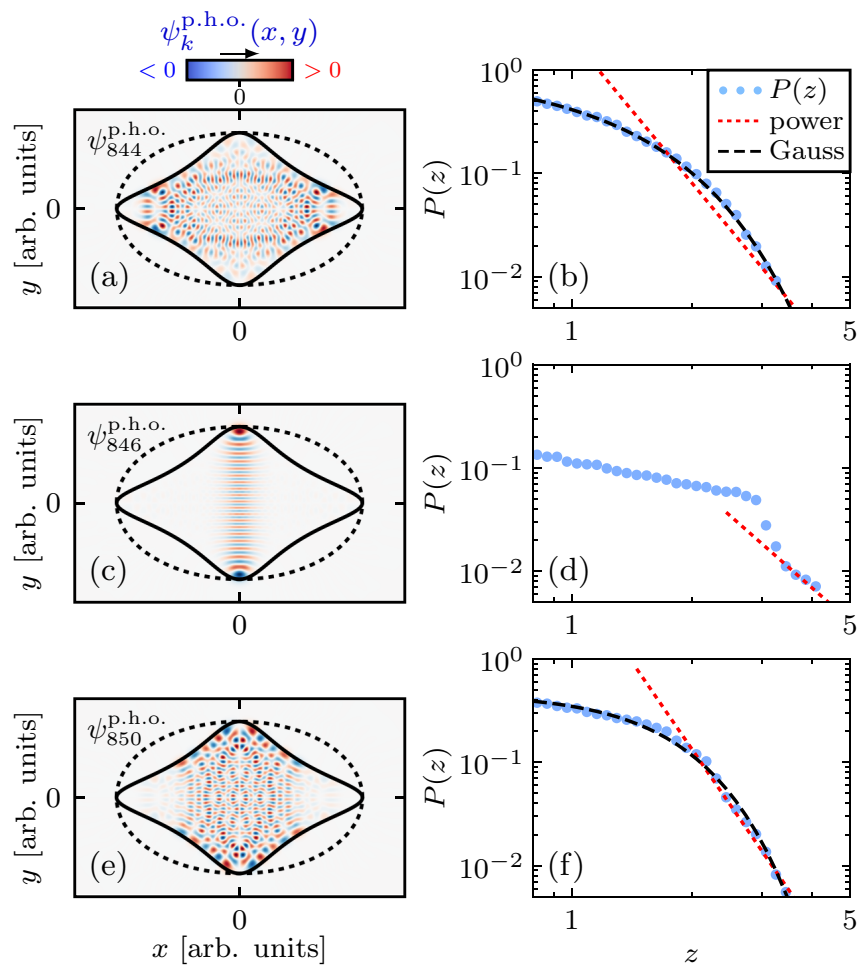

FIG. 7. Eigenstates of perturbed anisotropic 2D harmonic oscillator. The left-hand panels are wave functions $\psi_{k}^{\text {ph.o. }}(x, y)$ (labeled $k=0,1,2, \ldots$ in increasing order of the corresponding eigenvalues). The right-hand panels are double logarithmic plots of amplitude distributions $P(z)$ (blue points). We also indicate Gaussian and power-law fits. In the wave-function plots, solid lines indicate the equipotential curve $V(x, y)=E^{(k)}$, and dotted elliptical lines indicate corresponding curves for the unperturbed potential. $(a, b)$ For the state $\psi_{844}^{\text {p.h.o. }}, P(z)$ has a Gaussian tail. (c, d) The state $\psi_{846}^{\text {p.h.o. }}$, resembling a 1D harmonic oscillator eigenstate, shows $P(z)$ features similar to that seen in integrable systems: a kink followed by an arguably power-law tail. (e, f) State $\psi_{850}^{\text {p.h.o. }}$ is an intermediate state, whose tail fits neither a power law, nor a Gaussian, particularly well. 
$\psi_{m n}^{\text {h.o. }}$ are products of the eigenfunctions of the 1D harmonic oscillator and have amplitude distributions with power-law tails. In Fig. 6(c) we illustrate the example $(m, n)=(141,121)$. Like the circular billiard, this dependence arises due to a combination of many inequivalent peaks in the wave functions. The "kink" in the power-law regime at $z \approx 3$, that separates two regimes with different power-law exponents, presumably stems from the product structure.

Generically, if the potential is modified, many eigenfunctions at higher energies look chaotic and have amplitude distributions with Gaussian tails. Typically, the phase space at higher energies is mainly chaotic, with small regular islands surrounding the short stable periodic orbits. Thus, in this regime, one typically encounters only a few regular eigenfunctions among many chaotic ones. In order to illustrate this observation, let us consider the weakly anharmonic potential $V(x, y)=x^{2}+(\lambda y)^{2}+\alpha x^{2} y^{2}$, with $\alpha=0.2$. In Figs. 7(a) and 7(b), we show an example of a chaotic eigenstate, whose amplitude distribution is Gaussian and whose eigenfunction is random-wave-like in a significant area of the classically accessible region. At nearly equal energy, we also find an example of a highly regular eigenfunction [Figs. 7(c) and 7(d)]; typically, such eigenfunctions have a large overlap with a small number of eigenstates of the nonperturbed model. The amplitude distribution typically shows power-law tails after a kink, much like the $|m, n\rangle$ themselves. Furthermore, there are "intermediate" eigenstates which have an extended wave function, but neither a power law nor a Gaussian fits well [Figs. 7(e) and 7(f)].

\section{CONTEXT AND CONCLUSIONS}

We have extended the study of amplitude distributions to many-body quantum systems. One context for this work is a growing appreciation that concepts from the field of singleparticle quantum chaos can be useful for many-body quantum systems [1-5,28,29,46,53-58]. A global study of all manybody eigenstates, such as the present one, is not historically common in condensed matter physics. The full eigenspectrum has gained importance only recently, due to intense interest in the dynamics of isolated systems, including thermalizationrelated questions [1-5] and many-body localization [59,60].

Our most striking result is the hint of a remarkable and unusual type of universality associated with integrable manybody systems: the coefficient distribution approaches a power law in the large-size limit. We have presented data and arguments to conjecture that this is a generic feature of multiple classes of integrable systems. Interestingly, we have shown that a number of regular single-particle billiards also show power-law tails in $P(z)$, although we do not claim this to be generic.

For nonintegrable many-body systems, away from the spectral edges, we have found Gaussian amplitude distributions, as expected. An interesting feature is the slight deviation from Gaussianity in 1D geometries. Gaussian behavior is a measure for the randomness of eigenstates and thus a characterization of nonintegrable behavior; in this respect it complements other eigenstate properties such as entanglement randomness [42,45], inverse PR [5,28,42,46], phase correlators [61], ETH scaling [50,62], etc. However, beyond its connection to chaos in eigenstates, we regard the coefficient distribution to be an object of basic importance in its own right.

This work raises a number of questions:

(1) Ground states of many-body systems are multifractal $[63,64]$. Multifractality is related to the moments of $P(z)$; hence in light of the present work one would like to investigate multifractality in the full spectrum. This requires modifying the definition in terms of size scaling, since there is no natural correspondence between eigenstates of different-sized systems. Appendix D provides some data on multifractality in a nonintegrable many-body system.

(2) The distributions for eigenstates near the spectral edge are clearly not Gaussian (Fig. 1), but it is unclear whether there is any generic behavior or a generic limiting distribution at large sizes.

(3) In (near-integrable) quantum billiards with a mixed phase space, the non-Gaussianity of $P(z)$ can sometimes be described by, e.g., modified Gaussians with position-dependent variance [31]. For many-body systems, characterizing the nonGaussianity of near-integrable eigenstates remains an open task.

(4) The basis dependence of the distributions is an open question. For example, in a mean-field basis [5] such as the eigenbasis of the $X X(\Delta=0)$ Hamiltonian, the integrable $X X Z$ chain has a high-kurtosis non-Gaussian amplitude distribution, similar to the distribution in the coordinate basis [Fig. 1(b)].

Each of these questions points to interesting directions for future study.

\section{ACKNOWLEDGMENT}

We thank A. Chandran, M. Heyl, I. Khaymovich, A. Lakshminarayan, C. R. Laumann, and L. Santos for discussions.

\section{APPENDIX A: COMMENTS ON QUANTUM INTEGRABILITY}

In classical mechanics, the notion of integrability is commonly understood to mean the presence of (at least) as many conserved quantities as the number of degrees of freedom ("Liouville-integrability"). In contrast, for quantum systems, there are a number of different notions of integrability, and it is possible to find exceptions to or inadequacies with most definitions. We briefly discuss here a few notions associated with integrability, so that the sense in which we have used the term is sufficiently clear.

Single-particle quantum billiard systems are called chaotic if the dynamics of the corresponding classical Hamiltonian system is chaotic. Conversely, a single-particle quantum system may be regarded as integrable or regular if the corresponding classical system has integrable ("regular") dynamics. When the integrability is broken, the system typically has a so-called mixed phase space, consisting of regions with regular motion and regions with chaotic motion. This is reflected in the quantum eigenstates which are typically concentrated either within the regular regions or the chaotic regions.

For quantum many-body systems, the situation is substantially more complicated $[15,16]$. Let us first consider systems where a large-size ("thermodynamic") limit is naturally defined. For example, this includes fermionic or bosonic systems, 
where the limit is defined by increasing the system size while keeping the density constant, and magnetic systems where the limit is defined by increasing the system size while keeping the magnetization density constant. In such cases, the Hilbert-space dimension increases exponentially with system size. A common notion of integrability is that, if the system can be "solved" with polynomial rather than exponential effort, then the system is integrable. Here, "solving" means finding the eigenvalues and eigenstates of the Hamiltonian. For example, for systems of noninteracting fermions or bosons, it is sufficient to find the eigenvalues and eigenstates of the single-particle problem; this allows construction of the manybody eigenstates. For systems solvable by the Bethe ansatz, the problem can be reduced to a polynomial number of nonlinear equations. In the simpler examples of the Bethe ansatz, such as the $X X Z$ chain, the number of equations is equal to the number of particles. For more complicated cases, such as those requiring a nested Bethe ansatz solution, the counting is more complicated, but the basic idea of polynomial solvability still applies.

The idea of polynomial solvability is closely related to the physical idea that an integrable system has a macroscopic number of conserved quantities. The number of conserved quantities scales polynomially (generally linearly) with the system size. The conserved quantities for noninteracting fermions or bosons are the occupancies of single-particle modes. For systems integrable only via the Bethe ansatz, the conserved quantities are often difficult to construct explicitly, although their existence is guaranteed.

It is interesting to note that the above notion of integrability relies on large-size scaling, and thus strictly speaking is not defined for a single fixed-size system, which is in sharp contrast to the single-particle billiard case. However, if a manybody Hamiltonian is integrable, then a "reasonably large" system will show Poissonian level statistics. While this is a phenomenological statement and not very rigorous, it is sufficient for many purposes, and we can thus consider the level statistics to provide an operational distinction between integrable and nonintegrable many-body systems. The advantage of this viewpoint is that one can discuss integrability in both single-particle quantum billiards and in many-body systems within the same framework. Note, however, that even for the single-particle case there are integrable systems not following Poissonian statistics; these are usually considered as "nongeneric."

There are various situations where the setup assumed here is not appropriate. For example, there are single-impurity problems which are solvable by Bethe ansatz, such as the Kondo model, the Anderson impurity model, and the interacting resonant-level model. In these cases, a constant-density scaling is not natural, as the impurity is localized in space. In addition, these models are generally integrable only for linearized bath dispersions, and the high-energy spectrum of the linearized models may not be very physical. Another unclear situation involves zero-dimensional models, such as the two-site Bose-Hubbard model. Although the solution of this model can be written in Bethe ansatz form, the ansatz does not reduce an exponential problem, because the Hilbert space has polynomial size to begin with (growing linearly with the particle number). In this work, we have ignored such special situations and restricted to many-body models where a clean and natural thermodynamic limit can be defined.

\section{APPENDIX B: ENTANGLEMENT ENTROPY, PARTICIPATION RATIO, AND DEVIATIONS FROM GAUSSIANITY}

In this appendix, we present data showing how the entanglement entropy (EE), the participation ratio (PR), and the deviation from Gaussianity (quantified using the KLD) are correlated.

We first present scatter plots (one data point for each eigenstate) allowing visualization of the degree of correlation. Figures 8(a)-8(d) show plots of PR versus KLD for all eigenstates, for the $X X Z$ chain with and without NNN coupling, and for the bosonic model with and without interaction. Analogously, we show in Figs. 8(e)-8(h) the correlation between the KLD and

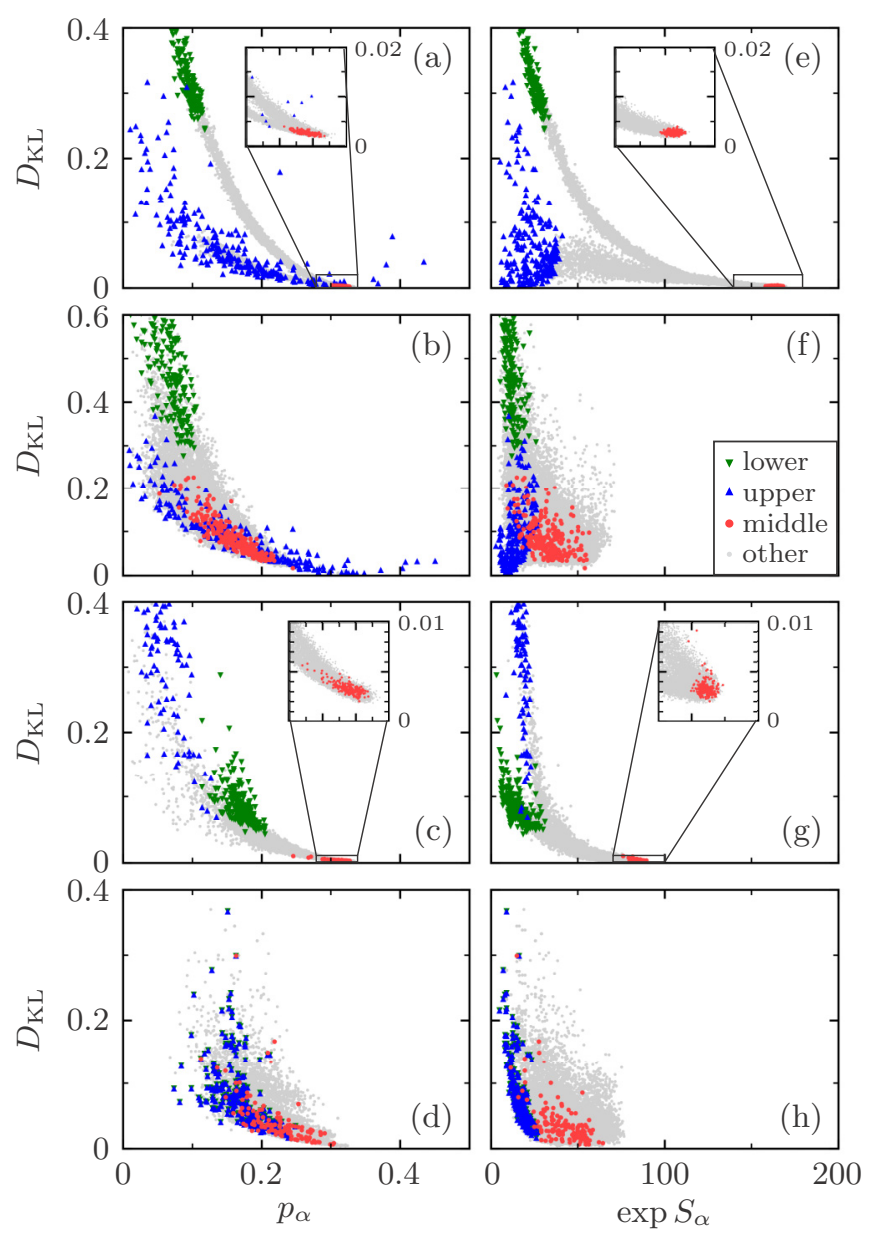

FIG. 8. Kullback-Leibler divergence plotted against participation ratio (a-d). We show data for (a) the nonintegrable $X X Z$ chain with NNN coupling $\left[\left(L, N_{\uparrow}\right)=(17,8)\right.$ and $\left.\lambda=1\right]$; (b) the integrable $X X Z$ chain (same size, $\lambda=0$ ); (c) the nonintegrable boson chain with Hubbard interaction $\left[\left(L, N_{\mathrm{b}}\right)=(13,6)\right.$ and $\left.\lambda=1\right]$; (d) the integrable boson chain without Hubbard interaction (same size, $\lambda=0$ ). (eh) Kullback-Leibler divergence against entanglement entropy with respect to left-right partition for the same systems as panels (a)-(d), respectively. In all cases, we have highlighted 250 states at the lower and upper edge of the spectrum and in the middle. 

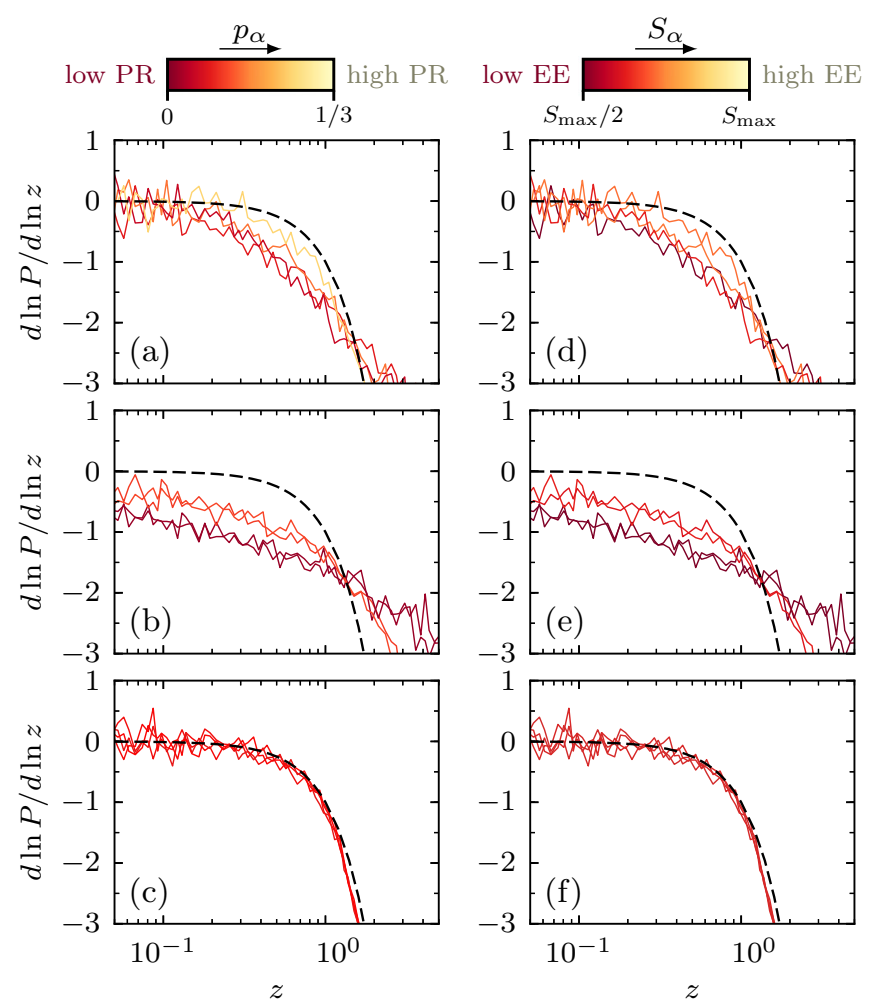

FIG. 9. Double-logarithmic derivative of the coefficient distribution for 4 individual eigenstates. (a, d) Integrable $X X Z$ chain $(\lambda=0)$, middle of spectrum, near $E=0$. (b, e) Nonintegrable $X X Z$ chain $(\lambda=$ 1), lower end of spectrum. (c, f) Nonintegrable $X X Z$ chain $(\lambda=1)$, middle of spectrum. Left column $(\mathrm{a}-\mathrm{c})$ : Colors (shading) indicate values $P_{\alpha}$ of the PR. The PR ranges from $P_{\alpha}=0$ to $P_{\alpha}=1 / 3$. (d-f): The same distributions, but with the color (shading) representing EE values $S_{\alpha}$. Values range from $S_{\max } / 2$ to $S_{\max }$, where $S_{\max }=8 \ln 2$ is the maximum possible entropy. The dashed curves indicate the Gaussian distribution.

$\exp \left(S_{\alpha}\right)$, where $S_{\alpha}$ is the EE with respect to a partition of the system into two connected parts of sizes $l$ and $l+1$, where $2 l+1=L$. As argued in Sec. II, there is strong correlation visible in the nonintegrable case [Figs. 8(a), 8(c), 8(e), and $8(\mathrm{~g})]$ : a large deviation from Gaussianity is associated with small PR and with small EE between spatial partitions. The correlation is less clear in the integrable cases [Figs. 8(b), 8(d), $8(\mathrm{f})$, and $8(\mathrm{~h})$ ], similar to previous findings for the correlation between EE and PR in eigenstates [42].

Next, in Fig. 9 we plot the double-logarithmic derivative of the coefficient distributions for some of the individual eigenstates. We have plotted the distributions of $c_{\gamma}^{(\alpha)}$ of four individual representatives close to the designated part of the spectrum. The chosen states are the highest-PR, lowest-PR, highest-EE, and lowest-EE eigenstates within each group of 250 eigenstates.

Comparing the curves, we observe a clear correlation between the shape of the distribution and the PR and EE: The curves that lie closest to the Gaussian, e.g., for the nonintegrable model in the middle of the spectrum [Figs. 9(c) and 9(f); cf. Figs. 8(b) and 8(f)] are high-PR and high-EE states, as seen from the coloring (shading). The other (flatter, more power-law like) curves are low-PR and low-EE states. For the nonintegrable model, the eigenstates close to the lower edge of the spectrum show integrable behavior. This situation, shown in Figs. 9(b) and 9(e) is very similar to generic eigenstates in the integrable system [Figs. 9(a) and 9(d)]. On the other hand, in the bulk of the nonintegrable spectrum [Figs. 9(c) and 9(f)], all eigenstates have nearly Gaussian coefficient distributions, and this is also reflected by the small variation in EE and PR. With the KLD as a measure of distance to Gaussian, these observations are consistent with those of Figs. 8(a), 8(b), 8(e), and $8(f)$.

\section{APPENDIX C: QUANTUM BILLIARDS: ANALYTICAL OBSERVATIONS}

In this appendix, we provide some analytical results on amplitude distributions for the single-particle ("quantum billiard") systems described in Sec. IV, namely, the square billiard and the circular billiard.

\section{Square billiard}

For the billiard in a square $\{(x, y) \mid 0 \leqslant x, y \leqslant 1\}$ (so that $\mathcal{A}=1$ ) with Dirichlet boundary conditions, the wave functions are given by

$$
\psi_{m n}^{\square}(x, y)=2 \sin (m \pi x) \sin (n \pi y)
$$

with eigenenergies $\pi^{2}\left(m^{2}+n^{2}\right)$. The coefficient distribution is independent of $m$ and $n$, so we choose the ground state $m=n=1$ for simplicity and without loss of generality.

We present a derivation of $P(z)$ as the $z$ derivative of the area of the region defined by $\psi_{11}(x, y)<z$. We first simplify the problem by studying the function $f(x, y)=\cos x \cos y$ (with $|x|,|y| \leqslant \pi / 2)$, which is a scaled and shifted version of the wave function $\psi_{11}(x, y)$. The region defined by $f(x, y)>c$ encloses an area $A(c)$ complementary to the one we desire to compute. Considering the area in the first quadrant $(x \geqslant$ $0, y \geqslant 0)$ only, we find

$$
\frac{1}{4} A(c)=\int_{0}^{\arccos c} d y \arccos (c / \cos y),
$$

using that the boundary is given by $x=\arccos (c / \cos y)$, and $y$ runs from 0 to $\arccos c$. Substitution $w=\cos y$, such that $d y=-d w / \sqrt{1-w^{2}}$, yields the integral

$$
\frac{1}{4} A(c)=\int_{c}^{1} \frac{d w}{\sqrt{1-w^{2}}} \arccos (c / w)
$$

The solution to our initial problem, namely, the size of the level set defined by $z=\psi_{11}(x, y)$, is proportional to the derivative of $A(z / 2)$, with a scaling factor $1 / \pi^{2}$. By computation of the derivative of Eq. (C3), we obtain

$$
P(z)=-\frac{1}{\pi^{2}} \frac{d}{d z}[A(z / 2)]=\frac{2}{\pi^{2}} K\left[\sqrt{1-(z / 2)^{2}}\right],
$$

where $K(k)=\int_{0}^{1}\left(1-t^{2}\right)^{-1 / 2}\left(1-k^{2} t^{2}\right)^{-1 / 2} d t$ denotes the complete elliptic integral of the first kind.

From this expression, we find the approximate behavior $\sqrt{2 / \pi^{2} z}$ near the maximum value $z=2$, i.e., a scaling $\sim z^{-\gamma}$ with $\gamma=\frac{1}{2}$. There is however no extended power-law behavior. Equation (C4) also shows that the coefficient distribution diverges for $z \rightarrow 0$. 


\section{Circular billiard}

For the circular billiard of radius 1 (with $\mathcal{A}=\pi$ ), the eigenstates of the Hamiltonian $H=-\nabla^{2}$ are given in polar coordinates $r, \phi$ by

$$
\psi_{m n}^{\circ}(\vec{r})=\mathcal{N}_{m n} J_{m}\left(j_{m n} r\right) \cos (m \phi),
$$

where $J_{m}$ is the Bessel function of the first kind of integer order $m \geqslant 0, j_{m n}$ is the $n$th zero of this function ( $n>0$ integer), and

$$
\mathcal{N}_{m n}^{2}= \begin{cases}2 /\left[\pi J_{m-1}\left(j_{m n}\right)^{2}\right] & (m>0) \\ 1 /\left[\pi J_{1}\left(j_{0 n}\right)^{2}\right] & (m=0)\end{cases}
$$

is the normalization factor. The energy eigenvalue of this state is $j_{m n}^{2}$.

The amplitude distribution $P(z)$ has a power-law tail when the radial quantum number $n$ is large, i.e., the eigenfunction has many oscillations in the radial direction. The method of obtaining $P(z)$ can be illustrated using Figs. 6(d) and 6(e). The value $P(z) d z$ is proportional to the area of the region where $|\psi(\vec{r})| \in(c, c+d c)$, with $c=z / \sqrt{\mathcal{A}}=z \pi^{-1 / 2}$, is satisfied. For a 1D function $\psi(r), P(z)$ is thus given by the sum over $1 /\left|\psi^{\prime}\left(r_{i}\right)\right|$ over all solutions $\psi\left(r_{i}\right)=c$.

As already addressed in Sec. IV, Figs. 6(d) and 6(e) also visualize two distinct regimes: For small $z,|\psi(r, 0)|=c=$ $z \pi^{-1 / 2}$ has a fixed number of solutions, and the coefficient distribution is thus determined by the derivatives. For larger $z$, the coefficient distribution is also affected by the number of solutions, which gradually decreases if $z$ is increased. The boundary between these regimes is the value of the smallest local maximum of $|\psi|$.

We will now focus on the limit of $m=0$ and large $n$. For large arguments $x$, the Bessel function $J_{m}(x)$ behaves as an oscillatory function with amplitude $\sim x^{-1 / 2}$. More specifically [see Eq. (9.2.1) of Ref. [65]],

$$
J_{m}(x)=\sqrt{\frac{2}{\pi x}}\left[\cos \left(x-m \frac{\pi}{2}-\frac{\pi}{4}\right)+O\left(|x|^{-1}\right)\right] .
$$

Given that $n$ is large, the argument $j_{0 n} r$ in the Bessel function is large except for small $r$. The contribution from $r \rightarrow 0$ is suppressed due to the geometry (Jacobian of polar coordinates); therefore it is reasonable to use the above large- $j_{0 n} r$ approximation. Thus

$$
\begin{aligned}
\psi_{0 n}^{\circ}(\vec{r}) & \approx \sqrt{\frac{1}{\pi J_{1}\left(j_{0 n}\right)^{2}}} \sqrt{\frac{2}{\pi j_{0 n} r}} \cos \left(j_{0 n} r-\frac{\pi}{4}\right) \\
& \approx \sqrt{\frac{1}{\pi r}} \cos \left(n \pi r-\frac{\pi}{4} r-\frac{\pi}{4}\right),
\end{aligned}
$$

where we have used $j_{0 n} \approx\left(n-\frac{1}{4}\right) \pi$ and $\pi J_{1}\left(j_{0 n}\right)^{2} \approx 2 / j_{0 n}$ according to approximation (C7).

Small $z$-We first derive the behavior of $P(z)$ of $\psi_{0, n}^{\circ}$ for small $z$. For $z=0$, we sum over all contributions where the wave function intersects zero. For this purpose, we find the derivative

$$
\begin{aligned}
\psi_{0 n}^{\circ}(r)= & -\frac{1}{2} \pi^{-1 / 2} r^{-3 / 2} \cos \left(n \pi r-\frac{\pi}{4} r-\frac{\pi}{4}\right) \\
& -r^{-1 / 2}\left(n-\frac{1}{4}\right) \pi^{1 / 2} \sin \left(n \pi r-\frac{\pi}{4} r-\frac{\pi}{4}\right)
\end{aligned}
$$

with respect to $r$. Let us label the zeros of $\psi_{0 n}$ by $r_{k}=j_{0 k} / j_{0 n} \approx$ $\left(k-\frac{1}{4}\right) /\left(n-\frac{1}{4}\right)$ with $k=1, \ldots, n$. At $r=r_{k}$, the cosine term vanishes and the sine term is of unit magnitude, so that we find

$$
\begin{aligned}
\left|\psi_{0 n}^{\circ \prime}\left(r_{k}\right)\right| & =\sqrt{\pi / r_{k}}\left(n-\frac{1}{4}\right) \approx \sqrt{\pi}\left(n-\frac{1}{4}\right)^{3 / 2}\left(k-\frac{1}{4}\right)^{-1 / 2} \\
& \approx \sqrt{\pi n^{3} / k}
\end{aligned}
$$

Summing over all zeros, the contribution to the coefficient distribution becomes (in approximation)

$$
2 \sum_{k=1}^{n} 2 \pi r_{k} /\left|\psi_{0 n}^{\prime}\left(r_{k}\right)\right| \sim \sum_{k=1}^{n} \frac{k}{n} \frac{k^{1 / 2}}{n^{3 / 2}}=\sum_{k=1}^{n} \frac{k^{3 / 2}}{n^{5 / 2}} \sim 1 .
$$

From this result, we deduce that the coefficient distribution has a finite value near 0 . We find the form $P(z) \approx \alpha+\beta z^{2}$ for small $z$ with $\alpha=\frac{8}{5} \sqrt{\pi}$ and $\beta>0$. The vanishing of the linear term in $z$ can be understood from a symmetry argument on the coefficient distribution of $\psi_{0 n}$, rather than of $\left|\psi_{0 n}\right|$.

Large $z$-When $z$ is larger than the smallest maximum, the number of intersections defined by $\psi_{0 n}(r)=c=z \pi^{-1 / 2}$ depends on the value of $z$. The maxima of $\left|\psi_{0 n}(r)\right|$ are found at $r_{k}^{\prime} \approx\left(k+\frac{1}{4}\right) /\left(n-\frac{1}{4}\right)$ and are characterized by $\left|\psi_{0 n}\left(r_{k}^{\prime}\right)\right| \approx$ $\sqrt{1 / \pi r_{k}^{\prime}} \approx \sqrt{n / k \pi}$. The number of intersections is twice the number of maxima with $\left|\psi_{0 n}\left(r_{k}^{\prime}\right)\right| \geqslant z$, i.e., $2 k_{\max }$ with $k_{\max }=$ $\left\lfloor n / \pi z^{2}\right\rfloor$. At the intersection points the derivatives are also roughly of the order $n^{3 / 2} k^{-1 / 2}$ (assuming that the sine term dominates, which is true for $r \gg 1 / n$, i.e., almost all $k$ except the smallest ones). Then, performing a similar summation as above, we obtain

$$
\begin{aligned}
2 \sum_{k=1}^{k_{\max }} 2 \pi r_{k}^{\prime} /\left|\psi_{0 n}^{\prime}\left(r_{k}^{\prime}\right)\right| & \sim \sum_{k=1}^{k_{\max }} \frac{k^{3 / 2}}{n^{5 / 2}} \sim k_{\max }^{5 / 2} / n^{5 / 2} \\
& \sim\left(n / c^{2}\right)^{5 / 2} / n^{5 / 2}=c^{-5} \propto z^{-5}
\end{aligned}
$$

This scaling is valid for $z \gtrsim 1(c \gtrsim \sqrt{1 / \pi} \approx 0.56)$, but the approximation becomes worse for large $z$, i.e., if $z \sim \sqrt{n}(c \sim$ $\sqrt{n / \pi}$ ). Numerical data (e.g., as shown in Fig. 6) agree with this finding: The coefficient distribution shows a power-law scaling $\alpha z^{-\gamma}$ with $\gamma \approx 5$, with a deviation of less than 0.05 for large $n$. (For example, $n=301$ yields $\gamma=-4.96 \pm 0.05$.) The multiplicative constant $\alpha$ is almost independent of $n$, because the coefficient distribution converges for $n \rightarrow \infty$ : In this limit, the zeros and maxima of $\left|\psi_{0 n}\right|$ become denser, but the envelope remains unaltered; cf. Eq. (C7).

For large $n$ and nonzero but small $m$, the numerical results show a similar scaling $z^{-\gamma}$, where $\gamma$ is close to 5. The $\cos (m \phi)$ argument does not alter the overall derivation outlined above.

\section{APPENDIX D: MULTIFRACTALITY}

The moments of the coefficient distribution are used to define multifractality of wave functions. It is known that ground states of many-body systems are generally multifractal $[63,64]$. We consider here extending this idea to the full spectrum. Following Ref. [63], we define multifractality in terms of the Rényi entropies obtained from the many-body 
wave-function coefficients $c_{\gamma}$,

$$
S_{\mathrm{R}}(q, D)=-\frac{1}{q-1} \ln \left(\sum_{\gamma=1}^{D}\left|c_{\gamma}\right|^{2 q}\right) .
$$

The summation runs from 1 to $D$, the Hilbert space dimension. Then the fractal dimensions are defined as

$$
\mathcal{D}_{q}=\lim _{D \rightarrow \infty} \frac{S_{\mathrm{R}}(q, D)}{\ln D} .
$$

Wave functions are multifractal if the fractal dimension $\mathcal{D}_{q}$ depends on the Rényi parameter $q$. They are simply fractal if $\mathcal{D}_{q}$ is a constant other than 1 . For Gaussian wave functions, they are expected to be constant at $\mathcal{D}_{q}=1$. Thus, we expect a difference in scaling behavior between the spectral edges and the midspectrum eigenstates. However, except for the ground state and the highest-energy state, it is not clear that there is a meaningful comparison between sizes: an eigenstate for $L=$ 13 cannot in general be unambiguously associated with an eigenstate of the $L=15$ system. The $D \rightarrow \infty$ limit in the definition is thus not a priori well defined.

In Fig. 10 we display scaled Rényi entropies for the nonintegrable spin chain defined in Eq. (1), with $\lambda=1$ and $\Delta=0.8$. We plot $S_{\mathrm{R}}(q, D) / \ln D$, for the ground state, for four states near the middle of the spectrum, and for the topmost (highest-energy) state. In each case, three different system sizes are compared.

The ground states and the topmost state of successive system sizes can be meaningfully compared, and the limit in the definition of $\mathcal{D}_{q}$ is unambiguous. Both these cases [Figs. 10(a) and $10(c)]$ have almost converged already; extrapolation will give
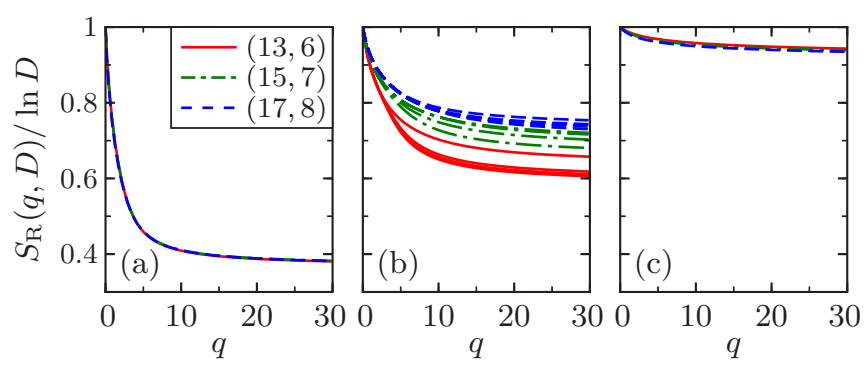

FIG. 10. Scaled Rényi entropies $S_{\mathrm{R}}(q, D) / \ln D$ for (a) the ground state, (b) four midspectrum states, and (c) the highest-energy eigenstate of the $X X Z$ model with NNN couplings. We compare three system sizes $\left(L, N_{\uparrow}\right)$, with $N_{\uparrow}=6,7,8$ up-spins in $L=2 N_{\uparrow}+1$ sites.

a $q$-dependent fractal dimension. Hence these non-Gaussian states are multifractal. For the ground state, this is consistent with the findings of Ref. [63].

For other eigenstates, the limiting procedure is not well defined, as explained above. Here, we simply take several eigenstates from near the center of the spectrum for each size [Fig. 10(b)]. The trend as the size increases is consistent with the expected nonfractal behavior $\left(\mathcal{D}_{q}=1\right)$. However, the approach toward $\mathcal{D}_{q}=1$ (assuming there is such an approach) is quite slow. Also, there are significant eigenstate-to-eigenstate fluctuations.

In summary, the data are consistent with the idea that the eigenstates are multifractal at the spectral edges and nonfractal in the middle of the spectrum, but the limit is not unambiguously defined and would be computationally challenging to characterize completely.
[1] J. M. Deutsch, Phys. Rev. A 43, 2046 (1991).

[2] M. Srednicki, Phys. Rev. E 50, 888 (1994).

[3] M. Rigol, V. Dunjko, and M. Olshanii, Nature (London) 452, 854 (2008).

[4] L. D’ Alessio, Y. Kafri, A. Polkovnikov, and M. Rigol, Adv. Phys. 65, 239 (2016).

[5] F. Borgonovi, F. M. Izrailev, L. F. Santos, and V. G. Zelevinsky, Phys. Rep. 626, 1 (2016).

[6] M. Shapiro and G. Goelman, Phys. Rev. Lett. 53, 1714 (1984).

[7] S. W. McDonald and A. N. Kaufman, Phys. Rev. A 37, 3067 (1988).

[8] R. Aurich and F. Steiner, Physica D 48, 445 (1991).

[9] R. Aurich and F. Steiner, Physica D 64, 185 (1993).

[10] B. Li and M. Robnik, J. Phys. A 27, 5509 (1994).

[11] F. Simmel and M. Eckert, Physica D 97, 517 (1996).

[12] T. Prosen, Phys. Lett. A 233, 332 (1997).

[13] A. Bäcker, in The Mathematical Aspects of Quantum Maps, Lecture Notes in Physics Vol. 618, edited by M. D. Esposti and S. Graffi (Springer, Berlin, 2003), pp. 91-144.

[14] A. Bäcker, Eur. Phys. J. Special Topics 145, 161 (2007).

[15] B. Sutherland, Beautiful Models: 70 Years of Exactly Solved Quantum Many-Body Problems (World Scientific, Singapore, 2004).

[16] J.-S. Caux and J. Mossel, J. Stat. Mech. (2011) P02023.
[17] O. Bohigas, M. J. Giannoni, and C. Schmit, Phys. Rev. Lett. 52, 1 (1984).

[18] M. V. Berry and M. Tabor, Proc. R. Soc. A 356, 375 (1977).

[19] G. Montambaux, D. Poilblanc, J. Bellissard, and C. Sire, Phys. Rev. Lett. 70, 497 (1993).

[20] D. Poilblanc, T. Ziman, J. Bellissard, F. Mila, and G. Montambaux, Europhys. Lett. 22, 537 (1993).

[21] T. C. Hsu and J. C. Anglès d'Auriac, Phys. Rev. B 47, 14291 (1993).

[22] D. A. Rabson, B. N. Narozhny, and A. J. Millis, Phys. Rev. B 69, 054403 (2004).

[23] A. R. Kolovsky and A. Buchleitner, Europhys. Lett. 68, 632 (2004).

[24] K. Kudo and T. Deguchi, J. Phys. Soc. Jpn 74, 1992 (2005).

[25] J. Karthik, A. Sharma, and A. Lakshminarayan, Phys. Rev. A 75, 022304 (2007).

[26] L. F. Santos, J. Math. Phys. 50, 095211 (2009).

[27] C. Kollath, G. Roux, G. Biroli, and A. M. Läuchli, J. Stat. Mech. (2010) P08011.

[28] L. F. Santos and M. Rigol, Phys. Rev. E 81, 036206 (2010).

[29] L. F. Santos, F. Borgonovi, and F. M. Izrailev, Phys. Rev. E 85, 036209 (2012).

[30] Y. Y. Atas, E. Bogomolny, O. Giraud, and G. Roux, Phys. Rev. Lett. 110, 084101 (2013). 
[31] A. Bäcker and R. Schubert, J. Phys. A 35, 527 (2002).

[32] E. J. Heller, Phys. Rev. Lett. 53, 1515 (1984).

[33] A. Bäcker, R. Schubert, and P. Stifter, J. Phys. A 30, 6783 (1997).

[34] M. V. Berry, J. Phys. A 10, 2083 (1977).

[35] C. E. Porter and R. G. Thomas, Phys. Rev. 104, 483 (1956).

[36] D. J. Luitz and Y. Bar Lev, Phys. Rev. Lett. 117, 170404 (2016).

[37] R. Mondaini and M. Rigol, Phys. Rev. E 96, 012157 (2017).

[38] R. Mondaini, K. R. Fratus, M. Srednicki, and M. Rigol, Phys. Rev. E 93, 032104 (2016).

[39] Y. Y. Atas and E. Bogomolny, J. Phys. A 50, 385102 (2017).

[40] S. Kullback and R. A. Leibler, Ann. Math. Statist. 22, 79 (1951).

[41] Here we separate the system in two spatial parts A and B of (nearly) equal size. Given a state $|\psi\rangle$, the entanglement entropy is $S=-\operatorname{Tr} \rho_{\mathrm{A}} \ln \rho_{\mathrm{A}}$, where $\rho_{\mathrm{A}}=\operatorname{Tr}_{\mathrm{B}}|\psi\rangle\langle\psi|$ is the reduced density matrix. The spatial partition is most relevant to the present study of amplitudes in the basis of real-space configurations.

[42] W. Beugeling, A. Andreanov, and M. Haque, J. Stat. Mech. (2015) P02002.

[43] V. Alba, M. Fagotti, and P. Calabrese, J. Stat. Mech. (2009) P10020.

[44] J. R. Garrison and T. Grover, Phys. Rev. X 8, 021026 (2018).

[45] L. Vidmar and M. Rigol, Phys. Rev. Lett. 119, 220603 (2017).

[46] L. F. Santos and M. Rigol, Phys. Rev. E 82, 031130 (2010).

[47] T. Tao and V. Vu, Adv. Math. 231, 74 (2012).

[48] H. H. Nguyen and V. Vu, Ann. Probab. 42, 146 (2014).

[49] C. Neuenhahn and F. Marquardt, Phys. Rev. E 85, 060101 (2012).

[50] W. Beugeling, R. Moessner, and M. Haque, Phys. Rev. E 89, 042112 (2014).
[51] W. Beugeling, R. Moessner, and M. Haque, Phys. Rev. E 91, 012144 (2015).

[52] R. Samajdar and S. R. Jain, J. Math. Phys. 59, 012103 (2018).

[53] M. Horoi, V. Zelevinsky, and B. A. Brown, Phys. Rev. Lett. 74, 5194 (1995).

[54] V. Zelevinsky, B. A. Brown, N. Frazier, and M. Horoi, Phys. Rep. 276, 85 (1996).

[55] V. V. Flambaum, F. M. Izrailev, and G. Casati, Phys. Rev. E 54, 2136 (1996).

[56] V. V. Flambaum and F. M. Izrailev, Phys. Rev. E 56, 5144 (1997).

[57] T. Guhr, A. Müller-Groeling, and H. A. Weidenmüller, Phys. Rep. 299, 189 (1998).

[58] M. Rigol and L. F. Santos, Phys. Rev. A 82, 011604 (2010).

[59] R. Nandkishore and D. A. Huse, Annu. Rev. Condens. Matter Phys. 6, 15 (2015).

[60] E. Altman and R. Vosk, Annu. Rev. Condens. Matter Phys. 6, 383 (2015).

[61] J. R. Armstrong, S. Åberg, S. M. Reimann, and V. G. Zelevinsky, Phys. Rev. E 86, 066204 (2012).

[62] M. Haque and P. McClarty, arXiv:1711.02360 [cond-mat.statmech] (2017).

[63] Y. Y. Atas and E. Bogomolny, Phys. Rev. E 86, 021104 (2012).

[64] D. J. Luitz, F. Alet, and N. Laflorencie, Phys. Rev. Lett. 112, 057203 (2014).

[65] M. Abramowitz and I. A. Stegun, Handbook of Mathematical Functions, Dover Books on Mathematics (Dover Publications, New York, 1972). 